\title{
The diagnostic significance of glucose-6-phosphate isomerase (G6PI) antigen and anti-G6PI antibody in rheumatoid arthritis patients*
}

\author{
Daren Yang ${ }^{1}$, Huinan $\mathrm{Ge}^{2}$, Jing Dong ${ }^{1}$, Xiongxiong Zhu², Gang Sun², Weiguo Ouyang ${ }^{2}$, \\ Linhui Wang ${ }^{3}$, Guoxing Zhang ${ }^{3}$ \\ ${ }^{1}$ Department of Clinical Laboratory, Suzhou Chinese Traditional Medicine Hospital, Suzhou, China \\ ${ }^{2}$ Department of Internal Medicine, Suzhou Chinese Traditional Medicine Hospital, Suzhou, China \\ ${ }^{3}$ Department of Physiology, Medical College of Soochow University, Suzhou, China \\ Email: zhangguoxing@suda.edu.cn
}

Received 30 May 2013; revised 30 June 2013; accepted 15 July 2013

Copyright (C) 2013 Daren Yang et al. This is an open access article distributed under the Creative Commons Attribution License, which permits unrestricted use, distribution, and reproduction in any medium, provided the original work is properly cited.

\begin{abstract}
Objective: To investigate whether glucose-6-phosphate isomerase (G6PI) antigen and anti-G6PI antibodies could be applied for the clinical diagnostic markers of rheumatoid arthritis (RA) and its associations with RA activity states. Methods: The levels of G6PI antigens and anti-G6PI Abs in sera from 176 RA patients in different states, 35 non-RA patients and 100 healthy donors and in synovia fluids from 33 patients and 11 non-RA patients were measured by ELISA. Results: The sensitivity and specificity of G6PI antigens in the RA patients were $75.0 \%$ and 93.3\%, respectively. The levels of serum G6PI antigens in $176 \mathrm{RA}$ patients were significantly higher than non-RA patients and the health controls. Especially, there was a significant difference between the active phase and the inactive phase in G6PI antigens levels. The levels of G6PI antigens in synovia fluid were also significantly higher in RA groups than in non-RA patients. With the values of the anti-G6PI Abs in sera, there were no marked differences among RA, non-RA patients and health controls. Also, there was no significant difference between the active phase and the inactive phase in RA patients. However, there were no significant differences of G6PI and anti-G6PI between RA patients and health controls in synovial fluid. Conclusions: G6PI is highly correlated with the activity states of RA, and could be applied for a clinical biomarker with high sensitivity and specificity for the diagnosis of RA.
\end{abstract}

*Funding: The study was supported by the Research Program of Soochow University No. Q413400111.

Disclosure statement: The authors have declared no conflicts of interest.
Keywords: Glucose-6-Phosphate Isomerase (G6PI); G6PI Antibody; Rheumatoid Arthritis (RA)

\section{INTRODUCTION}

RA is a common autoimmune inflammatory disease, characterized by chronic inflammation in the synovial membrane, cartilage and bone. Following the development of RA, hands, wrists, feet, and other small joints gradually become deformed, accompanied with skeletal muscle atrophy [1-4]. Based on the clinical observations, varieties of antigens and autoantibodies have been reported to be related to RA in patients. Reliable and specific diagnostic markers become more and more necessary to provide beneficial effects for clinical treatment with RA patients.

An increasing number of researchers focused on glucose-6-phosphate isomerase (G6PI) antigens and antibodies. In 1999, Matsumoto et al. firstly reported that $\mathrm{K} / \mathrm{BxNT}$ cell receptor transgenic mice model produced autoantibodies against G6PI, which was demonstrated to be arthritogenic when it was injected into normal mice [5]. Later, Schaller et al. demonstrated that increasing of anti-G6PI Abs in most of RA patients, suggested a linkage between animal model and human RA [6]. However, they also found that anti-G6PI Abs were not unique to patients with RA but were present in many patients with inflammatory arthritis, proposing a notion that immunebased inflammatory arthritis induces increases of antiG6PI Abs and G6PI/anti-G6PI immune complexes, which in turn influence proinflammatory cytokines release and involve in the development of inflammatory arthritis $[7,8]$. More recently, Fan et al. reported that human 
serum G6PI alone or in combination with anti-cyclic citrullinated peptide Abs (anti-CCP Abs) may improve the clinical diagnosis of RA [9].

However, several other observations did not support this conception [10-13]. Kassahn et al. reported that few human autoimmune sera were detected with G6PI [10]. Herve et al. found that G6PI was not a specific autoantigen in RA [12]. Matsumoto et al. also reported the low prevalance of anti-G6PI Abs in patients with RA [13]. Taken together, existing data still argue the practical usage of G6PI antigen or anti-G6PI Abs as clinical diagnostic markers for RA patients.

In the present study, we analyzed serum and synovia fluid from patients with different autoimmune diseases to evaluate G6PI antigen and anti-G6PI Abs, and compared the expressions of G6PI antigen and anti-G6PI Abs in RA patients with different activity states. Our results show that G6PI antigen has high sensitivity and specificity, and is highly correlated with the activity states in RA patients, but not anti-G6PI Abs.

\section{PATIENTS AND METHODS}

\subsection{Patients Recruited for Evaluation of G6PI Antigen and Anti-G6PI Abs}

From 2006 to 2009, inpatients and outpatients were recruited for the present study from Suzhou Chinese Traditional Medicine Hospital and Shanghai East Hospital. The research was in compliance with the Declaration of Helsinki and approved by the ethics committee of Soochow University. Informed consent was obtained from each patient. Diagnostic standard for RA patients was based on established clinical criteria [14]. RA patients include active stage $(\mathrm{n}=96)$ and inactive stage $(\mathrm{n}=80)$ (mean age $42.8 \pm 15.2$ years, range $18-94$, male $=36$, female $=140$ ). RA active stage diagnostic criteria is according to three items among the following: 1) joint swelling $\geq 3$; 2) joint tenderness $\geq 3$; 3) morning stiffness $\geq 45$ min. 4) average handgrip: male $\leq 192 \mathrm{mmHg}$, female $\leq 146 \mathrm{mmHg}$; 5) erythrocyte sedimentation rate: male $\geq 25 \mathrm{~mm} / \mathrm{h}$, female $\geq 30 \mathrm{~mm} / \mathrm{h}$. Otherwise patients were ascribed to inactive stage. Other autoimmune diseases patients include ankylosing spondylitis (AS) $(n=$ 10), systemic lupus erythematosus (SLE) $(\mathrm{n}=10)$, Sjogren syndrome (SS) $(\mathrm{n}=7)$, mixed connective tissue disease (MCTD) $(n=5)$, which are in line with the New York Revised Criteria for AS in 1984, The Revised Criteria for Classification of SLE in 1997, The Diagnostic Criteria for SS in 1992 (the European Union), Sharp Criteria for MCTD in 1972, respectively. Health control samples were collected from physical examination center of Shanghai East Hospital $(\mathrm{n}=100$, mean age $44.3 \pm$ 16.1 years, range $22-85$, male $=55$, female $=45$ ).

\subsection{ELISA for the Detection of G6PI Antigen}

G6PI antigen assay kits were purchased from R\&D System Co. Ltd. (Shanghai, China). Experiment procedure was according to the manufacture's instruction. In brief, add $50 \mu \mathrm{l}$ samples, standard and blank into the 96-well plate, incubate for 2 hours at $37^{\circ} \mathrm{C}$, wash the plate for five times, add $50 \mu$ Enzyme Conjugates to each well, incubate for 1 hours at $37^{\circ} \mathrm{C}$, wash the plate for five times, add $50 \mu \mathrm{l}$ Substrate Solution, incubate for $20 \mathrm{~min}$ utes at $37^{\circ} \mathrm{C}$ in dark place, add $50 \mu \mathrm{l}$ Stop Solution, measure absorbance at $490 \mathrm{~nm}$ wave length, $630 \mathrm{~nm}$ as reference wave length in microplate reader (Bio-Rad Laboratories, Inc, Shanghai, China, Model 680). According to the standard curve, calculating the concentration for each sample, G6PI $>0.25 \mu \mathrm{g} / \mathrm{ml}$ is regarded as positive. G6PI standard is provided by Shanghai Beijia Biochemistry Reagent Co. Ltd. (Shanghai, China).

\subsection{ELISA for the Detection of Anti-G6PI Abs}

Recombinant human G6PI antigen $(5 \mu \mathrm{g} / \mathrm{ml}$, generous gift from Sigma-Aldrich Co. Ltd. Shanghai, China) is added into 96-well microplate, sealed and incubated for overnight at $4^{\circ} \mathrm{C}$, wash the plate for 5 times, add 3\% BSA PBS buffer for 2 hours. Add $100 \mu \mathrm{l}$ samples (1:300 diluted with $0.1 \%$ BSA PBS buffer) to the well. Incubate for 2 hours at $37^{\circ} \mathrm{C}$; wash the plates for 5 times. Add 100 $\mu 1$ HRP-linked rabbit anti-human IgG (1:8000 dilution), incubate 20 minutes at $37^{\circ} \mathrm{C}$, wash the plates 5 times, add $100 \mu 1$ Substrates Solution, incubate at dark place for 20 minutes, add $100 \mu \mathrm{l}$ Stop Solution, measure absorbance at $450 \mathrm{~nm}$ wavelength.

\subsection{Statistical Analysis}

Concentrations of G6PI antigen and anti-G6PI Abs expressed as mean $\pm \mathrm{SD}$. Data analyses were performed by using Sigma Stat (SPSS, Chicago, IL) software. Due to the unmoral distribution of sera and synovial fluids, correction of t-test was used between two groups. LSD and S-N-K ANOVA were performed among three or more groups. $\mathrm{P}$ value $<0.05$ was considered statistical significant.

\section{RESULTS}

\subsection{Expression of G6PI Antigen in Sera}

The average concentration of G6PI in sera from RA is $4.34 \pm 8.65 \mu \mathrm{g} / \mathrm{ml}$, which is significantly higher than that from other autoimmune diseases $(0.07 \pm 0.07 \mu \mathrm{g} / \mathrm{ml}, \mathrm{P}<$ 0.01 ), and also higher than that from health control group $(0.34 \pm 1.30 \mu \mathrm{g} / \mathrm{ml}, \mathrm{P}<0.01)$. In RA group, the mean concentration of G6PI of active state patients is $7.61 \pm$ $10.69 \mu \mathrm{g} / \mathrm{ml}$, which is markedly higher than that of inactive state patients $(0.47 \pm 0.47 \mu \mathrm{g} / \mathrm{ml}, \mathrm{P}<0.01)$ 
(Table 1). Using the present ELISA method, we plot the receiver operating characteristic curve (ROC) of G6PI antigen (Figure 1). The ROC curve indicates that the best cut-off concentration for diagnosis of clinical RA is $0.25 \mu \mathrm{g} / \mathrm{ml}$. The sensitivity and specificity of the present method are $75.0 \%$ and $93.3 \%$ respectively.

\subsection{Expression of G6PI Antigen in Synovial Fluid}

In the synovial fluid, the concentration of G6PI from RA patients is $1.39 \pm 1.58 \mu \mathrm{g} / \mathrm{ml}$, which is significantly higher than that from other autoimmune diseases patients $(0.16 \pm 0.17 \mu \mathrm{g} / \mathrm{ml}, \mathrm{P}<0.01)$ (Figure 2(a)).

\subsection{Expression of Anti-G6PI Abs in Sera}

The mean optical density (OD) value of anti-G6PI abs in sera from RA group is $0.79 \pm 0.56$, in active RA is $0.87 \pm$ 0.63 , in inactive RA is $0.69 \pm 0.44$, in other autoimmune diseases is $0.58 \pm 0.25$ and in health control group is 0.70 \pm 0.46 , respectively. There is no significant difference

Table 1. Concentration of G6PI antigen in RA, other autoimmune diseases and health groups.

\begin{tabular}{|c|c|c|c|c|}
\hline \multirow[b]{2}{*}{ Group } & \multirow[b]{2}{*}{ Patients } & \multirow[b]{2}{*}{ G6PI $(\mu \mathrm{g} / \mathrm{ml})$} & \multicolumn{2}{|c|}{ G6PI positive rate } \\
\hline & & & $\begin{array}{l}\text { Patients } \\
\text { (n) }\end{array}$ & $\begin{array}{c}\text { Percentage } \\
(\%)\end{array}$ \\
\hline RA & 176 & $4.34 \pm 8.65^{*}$ & 132 & 75.0 \\
\hline Active RA & 96 & $7.61 \pm 10.69^{\#}$ & 84 & 87.5 \\
\hline Inactive RA & 80 & $0.47 \pm 0.47$ & 48 & 60.0 \\
\hline $\begin{array}{c}\text { Other autoimmune } \\
\text { diseases }\end{array}$ & 35 & $0.07 \pm 0.07$ & 0 & 0.00 \\
\hline Health & 100 & $0.34 \pm 1.30$ & 9 & 91.0 \\
\hline
\end{tabular}

G6PI concentrations are expressed as mean $\pm \mathrm{SD} .{ }^{*} \mathrm{P}<0.01$ as compared with other autoimmune diseases and health; ${ }^{\#} \mathrm{P}<0.01$ as compared with inactive RA.

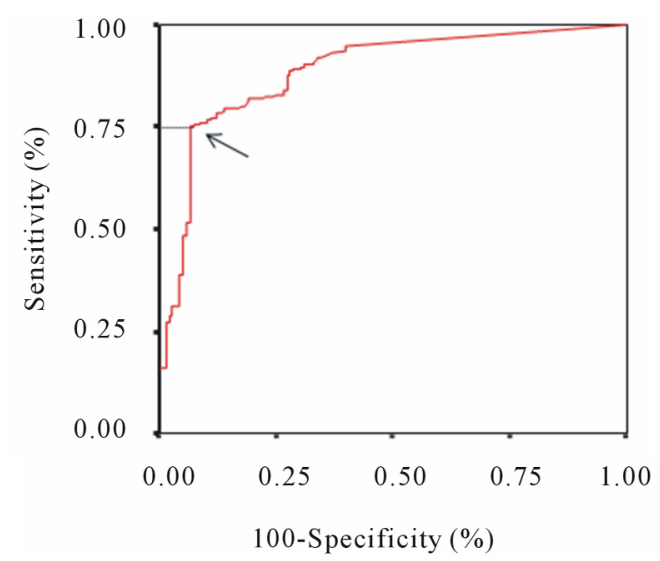

Figure 1. Receiver operating characteristic curve of G6PI ELISA. 100 health control and 176 rheumatoid arthritis sera were used to plot the curve. Arrow indicates the cut-off point. among RA, other autoimmune diseases and health control groups, also there is no significant difference between active and inactive groups in RA (Table 2).

\subsection{Expression of Anti-G6PI Abs in Synovial Fluid}

The mean OD value of anti-G6PI abs in synovial fluid from RA group is $0.73 \pm 0.52$, in other autoimmune diseases is $0.66 \pm 0.41$, there is no statistical difference between these two groups (Figure 2(b)).

\section{DISCUSSION}

In the present study, we clearly demonstrate that G6PI antigen level is higher in RA patients than other autoimmune diseases patients and health control, it is also highly related with the RA states, and active phase shows higher levels of antigen than that of inactive phase. However, we could not found any difference of anti-G6PI Abs among all groups, suggesting by using the present method, G6PI could be applied as another biochemistry marker for RA besides rheumatoid factor (RF) and anticyclic citrullinated peptide antibodies (anti-CCP Abs).

RA is a kind of unknown etiology, attacking small joints of limbs as autoimmune disease, it is a worldwide, high disabling disease, so clarifying the cause and investigating the mechanism of RA will provide beneficial effects for the early diagnosis and guide treatment for RA patients. Recently, researchers argued whether G6PI and anti-G6PI Abs could be applied as clinical diagnostic markers of RA.

In 1968, it was firstly realized that G6PI was a key enzyme for glycolysis and gluconeogenesis in the study of non-spherocytic hemolytic anemia. As a kind of multifunctional proteins in cells, it could induce the bone marrow stem cells to become different from mononuclear cells and B cell to become different from plasmacytes [15]. Yu et al. reported G6PI regulated matrix metalloproteinase-3 (MMP-3), and increased the expression of MMP-3 in the synovial cells and endothelial cells in RA patients, and promoted bone destruction. It was speculated

Table 2. OD value of anti-G6PI Abs in RA, other autoimmune diseases and health groups.

\begin{tabular}{ccc}
\hline Group & Patients (n) & Anti-G6PI Abs \\
\hline RA & 77 & $0.79 \pm 0.56$ \\
Active RA & 42 & $0.87 \pm 0.63$ \\
Inactive RA & 35 & $0.69 \pm 0.44$ \\
$\begin{array}{c}\text { Other autoimmune } \\
\text { diseases } \\
\text { Health }\end{array}$ & 23 & $0.58 \pm 0.25$ \\
\hline
\end{tabular}

OD indicates optical density; data are expressed as mean $\pm \mathrm{SD}$. 


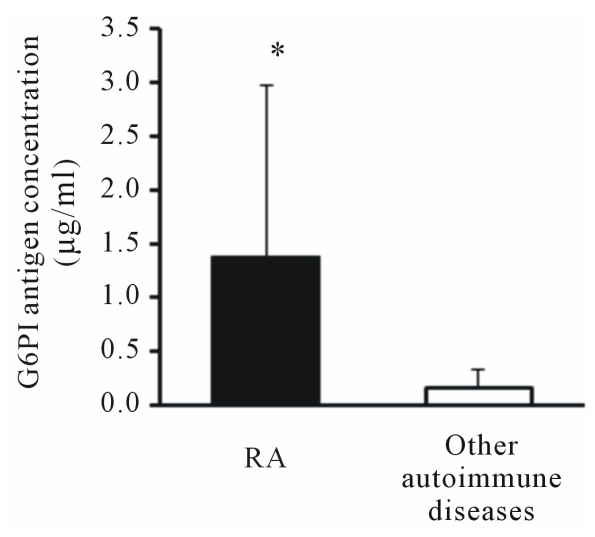

(a)

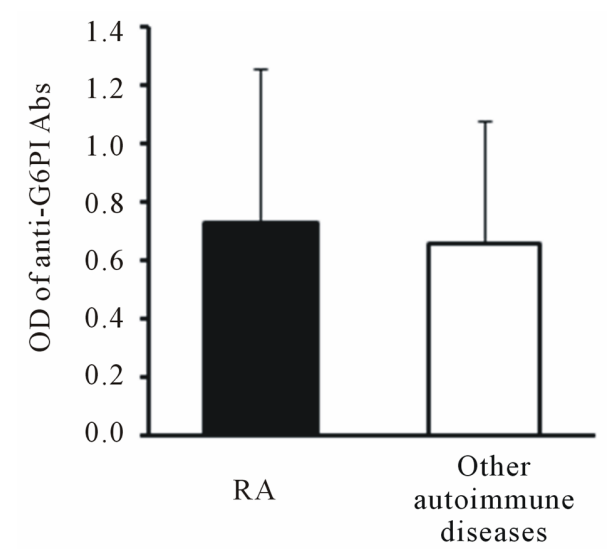

(b)

Figure 2. Concentration of G6PI antigen and anti-G6PI Abs in synovial fluids. (a) Concentration of G6PI antigen in synovial fluids from RA $(\mathrm{n}=33)$ and other autoimmune diseases $(\mathrm{n}=11) .{ }^{*} \mathrm{P}<0.01$ vs other autoimmune diseases group; (b) OD value of anti-G6PI Abs in synovial fluids from RA $(\mathrm{n}=33)$ and other autoimmune diseases at $450 \mathrm{~nm}$ wavelength.

that G6PI might play a role in vascular proliferation, synovial proliferation and bone destruction in RA patients [16]. However, it was reported that few human autoimmune sera could be detected with G6PI [10]. Herve et al. reported that glucose-6-phosphate isomerase is not a specific autoantigen in rheumatoid arthritis [12]. Other study also showed that G6PI concentration in sera and synovial fluids was increased in inflammatory arthritic diseases [8]. Recently, it was reported that G6PI was a useful marker for diagnosis of RA patients; it is positively correlated with anti-G6PI Abs, G6PI-containing immune complexes, G6PI mRNA, anti-CCP Abs, RF, C-reactive protein and ESR, respectively [9]. The EILSA data showed high sensitivity and specificity for RA patients, revealing the present method is reliable. Our data also showed that expression of G6PI in sera and synovial fluids from RA patients is higher than that of other auto- immune diseases and health control, suggesting the diagnostic application of G6PI in clinical. Discrepancy among the different laboratory for expression of G6PI in human sera and synovial fluids may be due to the different patients included, detection methods and sample pretreatment. Further study still needs to compare the difference between each method and the specificity of G6PI including all kinds of arthritis patients.

The most interesting finding of the present study is expression of G6PI is highly correlated with RA activity state. All of active RA patients showed higher levels of G6PI both in sera and synovial fluids compared with inactive RA patients. These data suggested that G6PI at least played a role in the development of RA, through which mechanisms still need further investigation. The present data will provide guide message for treatment with clinical patients under different stages.

Most of the researchers focused on the expression of anti-G6PI Abs. Matsumoto et al. demonstrated that antiG6PI Abs produced in $\mathrm{K} / \mathrm{BxNT}$ cell receptor transgenic mice was arthritogenic when it was injected into normal mice [5]. Later, although Schaller et al. demonstrated an increase of anti-G6PI Abs in most of RA patients, they also found that anti-G6PI Abs were not unique to patients with RA but were present in many patients with inflammatory arthritis, suggesting the unspecificity of antiG6PI Abs for RA patients [7,8]. Matsumoto et al. also reported the low prevalance of anti-G6PI Abs in patients with RA [13]. Our present data also demonstrated that anti-G6PI Abs were not different among all groups, suggesting anti-G6PI Abs were not good markers for diagnosis of RA.

Taken together, our present study demonstrates that G6PI could be applied for a clinical biomarker with high sensitivity and specificity for the diagnosis of RA. The increased expression of G6PI is highly correlated with the activity states of RA. On the contrary, anti-G6PI has little clinical value for the diagnosis of RA.

\section{REFERENCES}

[1] Lacotte, S., Brun, S., Muller, S. and Dumortier, H. (2009) CXCR3, inflammation, and autoimmune diseases. Annals of the New York Academy of Sciences, 1173, 310-317. doi:10.1111/j.1749-6632.2009.04813.x

[2] Sherer, Y. and Shoenfeld, Y. (2006) Mechanisms of disease: Atherosclerosis in autoimmune diseases. Nature Clinical Practice Rheumatology, 2, 99-106. doi:10.1038/ncprheum0092

[3] Firestein, G.S. (2003) Evolving concepts of rheumatoid arthritis. Nature, 423, 356-361. doi:10.1038/nature01661

[4] Gabriel, S.E. and Michaud, K. (2009) Epidemiological studies in incidence, prevalence, mortality, and comorbidity of the rheumatic diseases. Arthritis Research \& Therapy, 11, 229. doi:10.1186/ar2669 
[5] Matsumoto, I., Staub, A., Banjoist, C. and Mathis D. (1999) Arthritis provoked by linked T and B cell recognition of a glycolytic enzyme. Science, 286, 1732-1735. doi:10.1126/science.286.5445.1732

[6] Schaller, M., Burton, D.R. and Ditzel, H.J. (2001) Autoantibodies to G6PI in rheumatoid arthritis: Linkage between an animal model and human disease. Nature Immunology, 2, 746-753. doi:10.1038/90696

[7] Schaller, M., Stohl, W., Tan, S.M., Benoit, V.M., Hilbert, D.M. and Ditzel H.J. (2005) Raised levels of anti-glucose6-phosphate isomerase IgG in serum and synovial fluid from patients with inflammatory arthritis. Annals of the Rheumatic Diseases, 64, 743-749. doi:10.1136/ard.2004.025502

[8] Schaller, M., Stohl, W., Benoit, V., Tan, S.M., Johansen, L. and Ditzel H.J. (2006) Patients with inflammatory arthritic diseases harbor elevated serum and synovial fluid levels of free and immune-complexed glucose-6-phosphate isomerase (G6PI). Biochemical and Biophysical Research Communications, 349, 838-845. doi:10.1016/j.bbrc.2006.08.105

[9] Fan, L.Y., Zong, M., Wang, Q., Yang, L., Sun, L.S., Ye, Q., Ding, Y.Y. and Ma, J.W. (2010) Diagnostic value of glucose-6-phosphate isomerase in rheumatoid arthritis. Clinica Chimica Acta, 411, 2049-2053. doi:10.1016/j.cca.2010.08.043

[10] Kassahn, D., Kolb, C., Solomon, S., Bochtler, P. and Illges, H. (2002) Few human autoimmune sera detect GPI. Nature Immunology, 3, 411-412. doi:10.1038/ni0502-411b

[11] Schubert, D., Schmidt, M., Zaiss, D., Jungblut, P.R. and
Kamradt, T. (2002) Autoantibodies against GPI and creatine kinase in rheumatoid arthritis. Nature Immunology, 3, 411. doi:10.1038/ni0502-411a

[12] Herve, C.A., Wait, R. and Venables, P.J. (2003) Glucose6-phosphate isomerase is not a specific autoantigen in rheumatoid arthritis. Rheumatology, 8, 986-988. doi: $10.1093 /$ rheumatology $/ \mathrm{keg} 271$

[13] Matsumoto, I., Lee, D.M., Goldbach-Mansky, R., Sumida, T., Hitchon, C.A., Schur, P.H., Anderson, R.J., Coblyn, J.S., Weinblatt, M.E., Brenner, M., Duclos, B., Pasquali, J.L., El-Gabalawy, H., Mathis, D. and Benoist, C. (2003) Low prevalence of antibodies to glucose-6phosphate isomerase in patients with rheumatoid arthritis and a spectrum of other chronic autoimmune disorders. Arthritis \& Rheumatism, 48, 944-954. doi:10.1002/art.10898

[14] Klippel, J.H., Crofford, L.J.C., Stone, J.H. and Weyand, C.M. (2001) Primer on the rheumatic diseases. 12th Edition, Arthritis Foundation, Atlanta.

[15] Kraus, A.P., Langston Jr., M.F. and Lynch, B.L. (1968) Red cell phosphoglycerate kinase deficiency. A new cause of non-spherocytic hemolytic anemia. Biochemical and Biophysical Research Communications, 30, 173-177. doi:10.1016/0006-291X(68)90466-X

[16] Yu, F.L., Liao, M.H., Lee, J.W. and Shin W.L. (2004) Induction of hepatoma cells migration by phosphoglucose isomerase/autocrine motility factor through the upregulation of matrix metalloproteinase-3. Biochemical and Biophysical Research Communications, 314, 76-82. doi:10.1016/j.bbrc.2003.12.056 